The buffering power of overt socially supportive and unsupportive behaviors from the significant other on post-traumatic stress disorder individuals' emotional state

\begin{abstract}
Background and Objectives: Social support is one of the three strongest predictors of posttraumatic stress disorder (PTSD). In the present study, we aimed to assess the buffering power of overt socially supportive and unsupportive behaviors from the significant other, in a group with PTSD and a comparison group. Design and Methods: A total of 46 individuals with PTSD and 42 individuals with obsessive-compulsive disorder (OCD) or panic disorder (PD) completed diagnostic interviews and an anxiety-oriented social interaction with a significant other. Heart rate of participants was continuously measured during this interaction and overt social behaviors from the significant other were recorded on videotape and coded using a validated system. Results: Changes in heart rate in PTSD participants correlated negatively with changes in overt socially supportive behaviors from their significant other $(r$ from -.36 to $-.50, p$ $<.05$ ), while changes in overt unsupportive social behaviors from their significant other did not yield any significant correlation ( $r$ from -.01 to $.05, p>.05$ ). No such statistically significant association emerged in the group with OCD or PD ( $r$ from .01 to $-.27, p>.05)$. Conclusions: This study sustain the buffering power of overt supportive behaviors from the significant other on heart rate changes in PTSD.
\end{abstract}

Word count: 197

Keywords: Posttraumatic Stress Disorder; Heart Rate; Overt Supportive and Unsupportive Social Interactions; Panic Disorder; Obsessive-compulsive Disorder. 


\section{The buffering power of overt socially supportive and unsupportive behaviors from the significant other on post-traumatic stress disorder individuals' emotional state}

Posttraumatic stress disorder (PTSD; APA, 2013) has been extensively studied in relation to social support (Brewin, Andrews, \& Valentine, 2000; Guay, Billette, \& Marchand, 2006; Ozer, Best, Lipsey, \& Weiss, 2003). Two meta-analyses showed that social support was one of the three strongest predictors of the development and maintenance of PTSD and its symptoms $(r=$ .28 and $r=.40$; Brewin et al., 2000; Ozer et al., 2003).

A number of predominant theoretical models of PTSD sustained the influence of social support in the development and maintenance of PTSD (see Foa, Steketee, \& Rothbaum, 1989; Foy, Osato, Houskamp, \& Neuman, 1992; Horowitz, 1986; Jones \& Barlow, 1990; Joseph, Williams, \& Yule, 1997; Keane, Zimering, \& Caddell, 1985). More precisely, social support has been proposed to have an impact on PTSD via its influence on the individual's interpretation of the traumatic event (Joseph et al., 1997; Williams \& Joseph, 1999). In addition, actual or perceived support received from significant others, or lack thereof, is thought to lower or exacerbate stress levels, as well as have an impact on the individual's emotional state (e.g. fear, panic, grief, guilt, and shame) and coping strategies (e.g. degree of avoidance of thoughts and behaviors). In other words, social support was conceptualized as a buffer (Buffering hypothesis: Cohen \& Wills, 1985; Lakey \& Cohen, 2000) of individuals' reactions to stress. However, there is a continued need for empirical data to support these conceptualizations.

An objective index of individuals' emotional state or reactions to stress can be provided by physiological measures. Physiological responsiveness is clearly a crucial dimension of PTSD (see Nachar, Lavoie, Marchand, O'Connor, \& Guay, 2014; Pole 2006, 2007). Indeed, individuals

with PTSD have persistently showed increased sympathetic responses or reduced 
parasympathetic activity (Hopper, Spinazzola, Simpson, \& van der Kolk, 2006; Sack, Hopper, \& Lamprecht, 2004) to both trauma-related cues and unconditioned stimuli, as measured by changes in heart rate (Lang \& McTeague, 2009).

Received social support in a stressful situation is thought to attenuate heart rate in that context. In their meta-analysis, Uchino, Cacioppo, \& Kiecolt-Glaser (1996) concluded that the presence of an emotionally supportive other was associated with reduced heart rate. More specifically, when social support and stressors were manipulated in a laboratory (i.e. confederates or friends acted supportive, provided emotional support and problem-solving support), the relationship between social support and heart rate in non-clinical samples was characterized by a moderate effect size of .28 (Uchino et al., 1996). Does this apply to individuals with PTSD? Results from our own previous study revealed that a trauma-oriented social interaction with a significant other was accompanied by increased heart rate, and this outcome was correlated with the severity of PTSD symptoms (see Nachar et al., 2014). More severe PTSD symptoms were associated with larger increases in heart rate when individuals spoke about their trauma to a significant other. Some evidence was found to support the relationship between increased heart rate and the interaction between perceived social support and PTSD symptoms. We also found that perceived unsupportive social interactions with the significant other were related to heart rate while talking about one's trauma. Perceived unsupportive social interactions influenced heart rate irrespective of psychological status (i.e., a main effect). Despite everything, our study addressed perceived social support and did not focus on trauma-oriented social interaction and the overt behaviors of the significant other during that interaction. Such a perspective would extend the results of our own study and of other research addressing the links between social support and PTSD. 
A limitation in the literature on social support and PTSD is thus the exclusive reliance upon global self-report measures of social support, leaving aside specific dimensions of anxiety such as associated heart rate and overt behavioral support processes. A multi-method strategy for measuring social support processes of individuals with a PTSD and their significant other would enrich our knowledge about the links between PTSD and social support in part by providing empirical evidence of what has been previously proposed on a theoretical level.

Although an approach measuring both perceived and received social support has been recommended (Wills \& Shinar, 2000), few studies on PTSD have used more than one method of evaluation, therefore limiting the extent of the results. In most studies, self-administered questionnaires were used to evaluate social support, and thus, mostly measured the perception of social support, or at least, what participants were willing to report. Observational measures of support behaviors exist (see Cutrona \& Suhr, 1992; Pash \& Bradbury, 1998), but are seldom used. Measures based on direct observation of dyadic behaviors are likely to provide information about social processes that a self-administered questionnaire could not supply (Wills \& Shinar, 2000). More precisely, direct observation would allow the assessment of verbal and non-verbal social support behaviors within dyads while simultaneously measuring heart rate.

In order to verify the specificity of these eventual links, another dimension to consider is the impact of social support on the emotional state of individuals with mental disorders other than PTSD, such as obsessive-compulsive disorder (OCD; APA, 2013) and panic disorder (PD; APA, 2013). Similarly to the traumatic memories in PTSD, obsessions and compulsions and panic attacks cause marked anxiety in, respectively, OCD and PD (APA, 2013).

Regarding the links between social support and OCD, one study revealed that more than $90 \%$ of immediate family members (parents or spouses) report accommodating the individual with OCD in their rituals (Calvocoressi et al., 1999). Accommodation, which can be seen as a 
form of social support, was significantly associated with family dysfunction, attitudes of rejection toward the individual with OCD, and psychological distress (Calvocoressi et al., 1995). Although family distress, accommodation, and rejection were related to depression and anxiety within the family (Black, Gaffney, Schlosser, \& Gabel, 1998), they were not linked to the severity of the patient's OCD (Amir, Freshman, \& Foa, 2000). In another study by Renshaw, Chambless, and Steketee (2003) with a mixed sample of OCD $(\mathrm{N}=41)$ and PDA $(\mathrm{N}=26)$ participants, it was found that the more participants perceived their primary relative ( $76 \%$ were spouses) as critical of them, the less they improved following their psychological treatment. Again, perceived criticism was found to be unrelated to concurrent symptom severity. Finally, Steketee (1993) found that poor social and family functioning, and household interactions characterized by anger and criticism, as reported by the patient before the therapy, predicted fewer gains on OCD symptoms 9 months following the end of the treatment. Perceived low social support was associated with poor quality of life in patients with OCD (Hou, Yen, Huang, Wang, \& Yeh, 2010).

Little data on social support and Panic disorder (PD) with or without agoraphobia has been collected. In a study comparing agoraphobic to non-agoraphobic married women, the authors found that agoraphobic women perceived their spouse as being less supportive (Pyke \& Roberts, 1987). Another study evaluating agoraphobic women and their spouses showed that husbands were perceived as being more critical of their wives compared to non-clinical couples, although they were not perceived as less supportive than control husbands (Chambless et al., 2002). Individuals with PD also perceive their social network as being unsupportive (Batinic, Trajkovic, Duisin, \& Nikolic-Balkoski, 2009). A recent retroactive study has revealed that PD participants, compared to matched healthy controls, had lower levels of social support, in part characterized by a lack of social integration and a poorer quality of interpersonal relationships (Batinic et al., 2009). Finally, as reported for OCD, it was found that the more participants 
perceived their primary relative or significant other as critical of them, the less they improved following psychological treatment, even though perceived criticism was found to be unrelated to concurrent symptom severity at pre-test (Renshaw et al., 2003).

\section{Goals and hypotheses}

This study seeks to evaluate whether the heart rate of individuals with PTSD varies as a function of the overt socially supportive or unsupportive behaviors of their significant other in a trauma-oriented social interaction. This would contribute to increase our knowledge of the links between PTSD and social support. It would also contribute to extend previous results obtained with the use of self-report questionnaires. This study also aims to evaluate whether the same patterns of results could be found in a group of individuals with mental disorders other than PTSD, such as OCD and PD.

The following hypothesis can be emitted: changes in overt positive socially supportive and socially unsupportive behaviors from the significant other during a trauma-oriented social interaction will correlate significantly with the anxious individual's changes in heart rate. More precisely, heart rate will vary negatively with observed overt socially supportive behaviors from the significant other and positively with overt socially unsupportive behaviors from the significant other. We will also test whether the links between changes in overt socially positive and unsupportive behaviors from the significant other and changes in heart rate are generalizable to mental disorders other than PTSD, in the present case, a mixed group of individuals with PD or OCD.

\section{Methods}

\section{Participants}

We recruited participants through advertisements in newspapers and referrals to the Research Center of Institut Universitaire en Santé Mentale de Montréal [trans. Montreal 
University Institute of Mental Health] by psychiatrists and other health practitioners in the Montreal (Canada) metropolitan area. This center is a large mental health institute, and is well known by the mental health community for conducting research on mental disorders. All participants had to have PTSD, OCD, or PD as their primary diagnosis, and their spouse or significant other (e.g. a close one, other than a relationship partner) had to give their consent to participate in the study. Exclusion criteria were: being younger than 18 years old, presence of a substance use disorder (abuse or dependence), and past or present psychotic episode, bipolar disorder, eating disorder, somatoform disorder or organic mental disorder. Married participants and those currently involved in an intimate relationship with a history of conjugal violence with their actual partner were excluded, mainly to avoid exacerbating any domestic violence issues. Characteristics of the final sample are presented in Table 1.

\section{Procedure}

Upon arrival to the research center and after signing informed consent forms, participants were interviewed and completed a battery of questionnaires. Participants were evaluated using the Structured Clinical Interview for DSM-IV (SCID; First, Spitzer, Gibbon, \& Williams, 1995), which assesses mental disorders, including PTSD, OCD, and PD. A doctoral research assistant with extensive training in administering the SCID conducted all of the clinical interviews. The interview was also used to ensure that participants met all of the inclusion criteria. In addition to the clinical interview, participants were asked to complete questionnaires at home and to return them at the next session, where they were invited to speak about their trauma and PTSD, or OCD or PD, while simultaneously measuring their heart rates.

The local institutional ethical and scientific review board approved the study.

\section{Instruments}

The Social Support Interaction Global Coding System (SICS) 
The SICS (Crevier, Marchand, Nachar, \& Guay, 2013) is a validated behavioral observation system adapted for a dyad formed by an anxious individual and their significant other. This instrument assesses 10 interactional behaviors (i.e., verbal and non verbal) of the significant other. They are grouped under six positive individual dimensions (i.e. SICS-positive):

(a) problem description (i.e., details of the event and consequences), (b) validation (e.g., "I understand that it is difficult"), (c) listening (i.e., visual contact and nods), (d) expression of emotions (e.g., "I feel sad"), (e) positive solution proposals (e.g., "Maybe you could try to go out"), and (f) behavioral implication (i.e., express oneself, voice intonation, active gestures, and facial expressions), and four negative individual dimensions (i.e. SICS-negative): (a) dysphoria or depressed affect (i.e., crying), (b) withdrawal (i.e., looking away), (c) negative solution proposals (e.g., "You should stay home"), and (d) counter-validation (e.g., "You should stop complaining").

Trained raters assess participants on each interactional behavior using the video-recorded interactions. Raters use a 9-point scale from 1 (very low) to 9 (very high) according to the frequency and the intensity of behaviors. The 15-minute interaction (see 2.4.) is divided into three blocks of 5 minutes and a score is given for each dimension on every block. The total score of each dimension is represented by the mean of the three scores obtained on each block.

Interrater reliability (i.e., intraclass correlations and alpha), completed for the third of the sample, was very good in the current samples (PTSD group: $r=.84, p<.01$ and $\alpha=.91$; other anxiety disorder group: $r=.89, p<.01$ and $\alpha=.94$ ).

\section{ECG recordings and data extraction}

The raw electrocardiogram (ECG) signal was recorded from two bipolar tin electrodes placed on the chest, across both sides of the sternum, and a ground electrode placed on the left side of the abdomen derived from the standard Einthoven triangle. The signal was fed into a 
wireless bioelectric digital amplifier (CleveMed Medical Devices, Inc.: http://www.clevemed. com) with a band-pass between .10 to $30 \mathrm{~Hz}$, digitized continuously at a sampling rate of $256 \mathrm{~Hz}$. The wireless signal was transmitted via the Bioradio model 110 to a receiver connected to the USB 2.0 port of a Pentium PC. The Bioradio Capture Lite ${ }^{T M}$ software was used for recording the ECG signal. Thus, the raw signal was processed with g.RTanalyze software (Guger technologies, Graz, Austria: http://www.gtec.at) running on a MatLab/Simulink platform (version 7.0). Average heart rates were translated into beats-per-minute from the interbeat interval (in ms), calculated from an automatic R-wave peak detection for each period. Invalid samples, most likely due to movement artifact, were removed from the calculation after a visual inspection of each trial.

\section{Heart rate Assessment}

Heart rates were recorded during the second assessment session. First, participants were asked about their general physical health and medical condition. After this brief interview, participants were seated on a chair in a neutral room with a small table. The apparatus consisted of a programmable wireless physiological monitor attached to the participant's belt. In order to quantify arousal and activation, the average heart rate was recorded continuously across 4 periods. The first period (T1) consisted of a two minutes measure during which PTSD participants were instructed to sit still naturally, avoiding any effort. This period was designed to measure the heart rate of individuals when entering the laboratory. Two minutes was sufficient to obtain valid indices (see Sharpley, 1993). The second period (T2; control period) corresponded to the "neutral" part of the discussion, where participants and their significant other were instructed to discuss a non-anxious topic for 10 minutes, during which the mean heart rate was captured. Ten minutes was a long enough period to capture a mean heart rate that would act as a control index and to which a second mean would be compared. The third period (T3; anxiety-oriented 
discussion) consisted of an "active" 15 minute discussion, where participants were invited to speak with their significant other about their mental disorder, its impact, their coping strategies, the social support they receive, and how they see the future. Partners or significant others were invited to behave as naturally as possible during the interaction. This third period was divided in three five-minute segments (T3a, T3b and T3c) in order to examine the evolution of heart rate in participants and overt socially supportive and unsupportive behaviors from the significant other over the 15-minute period. The last period (T4) consisted of a two minute long resting measure, during which participants were again instructed to sit still. This period was designed to measure the heart rate of individuals when they had completed the task. Two minutes was sufficient to obtain valid indices (see Sharpley, 1993). Both the first and second discussions were recorded on videotape. All participants conformed to the given instructions.

\section{Data analysis}

\section{Distribution of variables and normality of data distributions.}

First, descriptive data for both the PTSD group $(N=46)$ and the OCD or PD group $(N=$ 42) on a number of potentially confounding variables, or variables that may be associated with changes in heart rate and that could need to be controlled for in subsequent regression models (e.g. age, gender, cigarette, psychopharmacological medication, current relational status, and annual income) were calculated. Descriptive data for both groups on the other variables at hand (e.g. SICS-positive and negative, mean heart rate in T1, T2, T3a to c, and T4) were also calculated. Means and standard deviations were performed for continuous variables while proportions were performed for categorical variables. In addition, to assess the distributions of continuous variables and the normality of data, we performed descriptive statistics and Kolmogorov-Smirnov tests. In order to explore the differences between both groups on all these variables, comparison tests were performed. 
Changes in heart rate as a function of changes in overt socially supportive and unsupportive behaviors from the significant other

In order to reach our first objective, linear regressions were performed with changes in mean heart rate as the dependent variable, changes in overt behaviors from the significant other as the independent variable (SICS +, SICS -) included in the second block, and potentially confounding variables as covariates included in the first block.

In order to do so, we first calculated indices of change in mean heart rate and socially supportive and unsupportive behaviors from the significant other during the anxiety-oriented social interaction. Indices of change (i.e. simple difference score) in mean heart rate were calculated by: (a) subtracting mean heart rate in T3a from mean heart rate in T3b, (b) subtracting mean heart rate in T3a from mean heart rate in T3c, and (c) subtracting mean heart rate in $\mathrm{T} 3 \mathrm{~b}$ from mean heart rate in T3c. Therefore, a negative value would reflect a decrease in mean heart rate while a positive value would reflect an increase in mean heart rate. The same subtractions were done for overt socially supportive and unsupportive behaviors from the significant other, as measured by the SICS. In order to assess a potentially confounding influence that might account for the heart rate difference between periods, such as differences in overall verbal production that may have elevated or decreased heart rate (e.g. Stein \& Boutcher, 1993), proportions of time during which participants with PTSD and OCD or PD were engaged in the act of speaking were assessed in T3a, T3b, and T3c and differences (i.e. simple difference score) were calculated between these three segments. Then, correlation analyses were performed to assess if changes from segment to segment (e.g. T3a, T3b, T3c) in verbal behavior were significantly associated with changes in heart rate and if they needed to be controlled for in subsequent regression models. Links between the other potentially confounding variables and changes in heart rate indices as defined earlier were assessed. More specifically, Student's t-tests were performed to 
verify differences in changes in heart rate indices between: (a) gender, (b) prescribed psychopharmacological medication or not, (c) currently smoking cigarette (versus not), (d) marital status (currently living in a relationship with a partner versus not). Correlation tests (i.e. Pearson's $r$ or Spearman's rho, depending on data distribution) were also performed between changes in heart rate indices and (a) age and (b) annual income.

\section{Correlation differences between groups}

In order to achieve our second objective of comparing both groups, we decided to perform tests of comparisons of correlations by calculating z-scores with their p-values.

The alpha level was set at .05 for all analyses unless otherwise specified.

\section{Results}

\section{Variables' distributions and normality of data distributions}

Descriptive data for both the PTSD group $(N=46)$ and OCD or PD group $(N=42)$ on a number of potentially confounding variables, or variables that may be associated with changes in heart rate and that could need to be controlled for in subsequent regression models, are presented in Table 1. Descriptive data for both groups on the other variables at hand are presented in Table 2.

PTSD group

All variables (e.g. see Tables 1 and 2) were normally distributed, except for annual income $(N=46, K . S .=.13, p=.04)$, SICS-positive $(N=45, K . S .=.13, p=.04)$, and SICSnegative distributions $(N=45, K . S .=.22, p<.01)$. No outlier was found in these variables. OCD or PD group

All variables (e.g. see Tables 1 and 2) were normally distributed except distributions of age $(N=42, K . S .=.18, p<.01)$, annual income $(N=42, K . S .=.16, p=.01)$, and SICS-negative $(N=39$, K.S. $=.22, p<.01)$. No outlier was found in these variables. 


\section{Between-group comparisons}

Chi-square tests $\left(\chi^{2}\right)$ were performed to assess differences in proportions between both groups on: (a) gender, (b) cigarette, (c) medication, and (d) current relational status. Because age, annual income, SICS-positive and SICS-negative were not normally distributed, Mann-Whitney U tests were performed. Student's t-tests were performed for mean heart rate in every period and segment.

Descriptive statistics for the sample and between-group comparison tests are presented in Table 1. No statistically significant difference was observed between the PTSD group and the other mental disorders group.

Mean heart rate across periods and segments, and between group comparison tests are presented in Table 2. Significant differences in mean heart rate emerged in T3 (effect-size $r=$ .26), Т3a (effect-size $r=.27)$, T3b (effect-size $r=.27)$, T3c (effect-size $r=.23)$, to- and T4 (effect-size $r=.24$ ) between groups, suggesting higher reactions to the anxiety-oriented social interaction with a significant other for the PTSD group.

Changes in heart rate as a function of changes in overt socially supportive and unsupportive behaviors from the significant other

PTSD group

Changes in heart rate and changes in overt social support behaviors within the anxietyoriented social interaction are presented in Table 3. Correlations between changes in mean heart rate within the trauma-oriented interaction and changes in overt behaviors from the significant other are presented in Table 4. Only changes in the positive dimension correlated with changes in heart rate from T3a to T3c (as seen on Table 4).

Regression analyses 
Regarding the regression analyses, the following results emerged: (a) for [T3b-T3a], changes in SICS-positive were not significantly associated with changes in heart rate $(\beta=-.21, p$ $=.20)$ when changes in proportion of verbal behavior were controlled for $\left(R^{2}=.20, F(2,41)=\right.$ 5.03, $p=.01$ ), (b) for [T3c-T3a], changes in SICS-positive were significantly associated with changes in heart rate $(\beta=-.38, p=.01)$ when variables such as changes in proportion of verbal behavior, medication, cigarette, and age were controlled for $\left(R^{2}=.44, F(5,38)=5.88, p<.01\right)$, and (c) for [T3c-T3b], changes in SICS + were significantly associated with changes in heart rate $(\beta=-.35, p=.02)$ when variables such as changes in proportion of verbal behavior, medication, and cigarette were controlled for $\left(R^{2}=.46, F(4,39)=8.21, p<.01\right)$.

OCD or PD group

Changes in heart rate and changes in overt social support behaviors within the anxietyoriented social interaction are presented in Table 3. Correlations between changes in mean heart rate within the anxiety-oriented interaction and changes in overt behaviors from the significant other are presented in Table 4. No statistically significant association was observed (as seen on Table 4).

No significant association emerged between "control" variables and changes in heart rate. Moreover, given the absence of a significant correlation between changes in heart rate and changes in overt support behaviors, no regression analysis was performed.

\section{Correlation differences between groups}

Correlation differences between groups are presented in Table 4. Only the correlations for [T3c-T3b] changes were statistically significant between both groups at hand.

\section{Discussion}

We aimed to evaluate whether heart rate in individuals with PTSD (APA, 2013), taken as an "objective" index of individuals" emotional state or anxiety, varies as a function of overt 
socially supportive and unsupportive behaviors from their significant other in a trauma-oriented social interaction, a situation that can be experienced as stressful and consequently trigger a cardiac arousal. This line of research may contribute to our knowledge of the links between PTSD and social support. We also aimed to evaluate whether the same pattern of results would be found in a group with mental disorders (APA, 2013) other than PTSD, such as OCD and PD. As expected, changes in heart rate in individuals with PTSD during a trauma-oriented social interaction correlated negatively with changes in overt socially supportive behaviors from the significant other. In other words, for individuals with PTSD, the increase in overt socially supportive behaviors from their significant other was associated with the attenuation of their heart rate to a stressful situation such as a trauma-oriented social interaction. However, changes in overt unsupportive social behaviors from their significant other did not yield any significant correlation with changes in heart rate for individuals with PTSD. Moreover, no statistically significant association emerged between changes in overt social behaviors from the significant other and changes in heart rate in individuals with OCD or PD.

In light what was presented in an earlier and related study (see Nachar, et al., 2014 for the results and a more detailed discussion), the trauma-oriented discussion seemed to first specifically trigger a significant elevation in heart rate in individuals with PTSD (i.e. from T2 to T3a), which might be indicative of a sympathetic activation or a parasympathetic withdrawal. This was then followed by a decrease in their heart rate (i.e. from T3a to b and c), which might be indicative of a parasympathetic activation (see Nachar, et al., 2014) or a sympathetic withdrawal. In other terms, a trauma-oriented social interaction can be experienced, at first, as stressful for individuals with PTSD, usually avoiding such a situation (APA, 2013; Foa \& Rothbaum, 1998; see Nachar et al., 2014). However, this arousal decreased as a function of time, as shown by the attenuation in participants' heart rate (see Nachar et al., 2014, for a discussion). In addition, other 
disorders such as OCD and PD did not show the same pattern of results. No elevation in heart rate was observed here and during the anxiety-oriented social interaction under study. These results could be explained by a main hypothesis that could be further tested and empirically assessed in the future: The anxiety-oriented discussion might be experienced as less stressful than the trauma-oriented discussion. Indeed, individuals with an OCD or a PD might have acquired distance regarding their disorder, preventing them from reacting with elevated heart rate, which is not the case of individuals with PTSD discussing their trauma (see Lang \& McTeague, 2009).

Social interactions can be a source of stress for individuals with a disorder such as PTSD (Laffaye, Cavella, Drescher, \& Rosen, 2008), in addition to the effects of discussing their trauma (see Nachar et al., 2014). However, in light of the present results, overt socially supportive behaviors from the significant other have been shown to be associated with an attenuation in heart rate or anxiety to a stressful situation such as a trauma-oriented social interaction with a significant other. More precisely, trauma-oriented social interaction with a significant other, contrary to anxiety-oriented social interaction (e.g. PD-oriented and OCD-oriented), first triggered a significant elevation in participants' heart rate (see Nachar et al., 2014). This elevation was followed by an attenuation, which occurred as a function of time, during the trauma-oriented social interaction (see Nachar et al., 2014). This attenuation in heart rate was revealed here to be associated with an increase in overt socially supportive behaviors from the significant other. As mentioned before, the mere presence of a supportive other (Uchino et al., 1996) has been associated with attenuated heart rate in the general population. Our results can be considered as an extension of what has been previously observed in the general population. Our results also add empirical evidence to what was conceptualized in earlier studies. Indeed, perceived as well as overt social support could act as a buffer to stress in individuals with a PTSD (Buffering Hypothesis: Cohen \& Wills, 1985; Joseph et al., 1997; Williams \& Joseph, 1999; see Nachar et 
al., 2014).

Correlations were found between changes in overt social support behaviors from the significant other and changes in heart rate of individuals with PTSD. However, contrary to what was expected, no such correlation was found between changes in overt socially unsupportive behaviors from the significant other and changes in heart rate of individuals with PTSD. Moreover, no such statistically significant association emerged in the group with OCD or PD. A sort of participation bias could explain this. Social interactions with the significant other were predominantly positive and supportive in our sample (see Table 2), especially considering that couples involved in an intimate relationship with a history of conjugal violence were excluded. Indeed, couples or dyads that are willing to participate in such an experiment usually have positive relations (Aron, Norman, Aron, McKenna, \& Heyman, 2000). Thus, this makes it difficult to assess the associations between overt socially unsupportive behaviors from the significant other and changes in heart rate. It also makes it difficult to compare the associations between heart rate and, respectively, overt socially supportive and unsupportive behaviors from the significant other.

Attenuation in heart rate within a trauma-oriented social interaction has been observed in participants with PTSD. This decrease was associated with an increase in overt socially supportive behaviors from the significant other. Regarding the group with OCD or PD, results did not yield to the same patterns of associations. These results particularly highlight the specificity of these associations for the group of PTSD. The mere presence of a significant other seemed to be associated with the highest heart rates (i.e. heart rate in T2) followed by an attenuation that varied irrespectively of overt socially supportive and unsupportive behaviors from the significant other. In line with what was previously presented, even if OCD or PD individuals perceptions of their primary relative or significant is associated with their improvement following psychological 
treatment, perceived criticism was found to be unrelated to concurrent symptom severity at pretest (Renshaw et al., 2003). In other words, social support processes may have a facilitating impact on the result of their treatment but do not necessarily moderate their reactions to stress or their symptoms.

Support derives from the social network of an anxious individual. Social support usually refers to positive and supportive social interactions (e.g., helping, encouraging, or caring). However, a growing number of researchers believe that unsupportive social interactions (e.g., criticizing, avoiding, yelling, blaming, or stigmatizing) form a distinct pattern of social support related to mental health (see Guay et al., 2011). For instance, the impact of unsupportive social interactions on psychological health appeared to be independent of the impact of supportive social interactions in a population of university students (Abbey, Abramis, \& Caplan, 1985), and strong in a sample of individuals with PTSD (Ullman \& Filipas, 2001). Results from our own study suggest that both supportive and unsupportive interactions may be highly relevant dimensions to consider in the study of the links between social support and PTSD (see Guay et al., 2011). As mentioned earlier, our sample being predominantly positive in terms of social support, the difference between supportive and unsupportive behaviors from the significant other could have hardly be assessed in relation to changes in heart rate. A larger sample would also have helped us to address differences between the impact of socially supportive behaviors and that of unsupportive behaviors from the significant other on heart rate changes in an anxietyoriented social interaction. Despite everything, we were able to observe differences in the associations between overt supportive and unsupportive social behaviors from the significant other on one hand, and heart rate, on the other.

Moreover, overt support behaviors, as for perceptions of social support, were associated with changes in heart rate. It would be interesting to extend the study of overt supportive and 
unsupportive behaviors (Wills \& Shinar, 2000) and their impact on mental health, when compared to perceptions of social support. It would enrich our understanding of the mechanisms of the impact of social support on mental health.

\section{Study Limitations}

As previously mentioned, a greater number of participants within the sample would have yielded more definitive conclusions. For instance, unsupportive social interactions may have been found to be associated with changes in heart rate were the sample size larger. Considering the wide array of variables that have an impact on heart rate changes, larger samples with multivariate covariance analyses would help draw a more precise portrait of the determinants of heart rate in a stressful situation. A larger sample would have also allowed us to address sex differences as they relate to social support for instance. Indeed, our sample (especially the group with PTSD) was predominantly female and the power to detect sex differences was low. Also, a number of the variables examined seemed to be interconnected. A larger sample would have also allowed us to separate participants with OCD or PD into two different groups in order to confidently test for differences. A longitudinal design would help us clarify the portrait of causality. Finally, a measure assessing the quality of the relationship between the participants and their significant others would have enriched our results.

In future studies, heart rate variability could be further assessed in order to better understand the effects of both divisions of the autonomic nervous system (parasympathetic and sympathetic activation and withdrawal during the social interaction at study). The effect of other potential moderators on the links between PTSD and heart rate, such as comorbidity namely with anxious disorders like social phobia and trauma recurrence (McTeague et al., 2009; 2010), could be assessed. Indeed, anxious disorders such as social phobia have been linked with specific patterns of heart rate in the past (see McTeague et al., 2009). Moreover, a tape assisted recall 
strategy could be used to provide qualitative information on the significant other's intention regarding his behavior during the discussion in order to better understand the associations between overt behaviors of their significant other on their heart rate changes (Barker \& Pistrang, 2002).

\section{Conclusion}

As an extension to what was previously found regarding perceived social support (see Nachar et al., 2014) and heart rate in individuals with PTSD, heart rate varied as a function of overt socially supportive behaviors from the significant other in a trauma-oriented social interaction. This was not the case with OCD and PD. Social support may operate differently in PD and OCD than in PTSD. Further studies are needed to clarify the patterns and impact of social support in anxiety disorders other than PTSD. Such studies could in turn orient future research, as it has been the case with PTSD.

\section{References}

Abbey, A., Abramis, D.J., \& Caplan, R.D. (1985). Effects of different sources of social support and social conflict on emotional well-being. Basic and Applied Social Psychology, 6, 111129. doi: $10.1207 / \mathrm{s} 15324834 \mathrm{basp} 0602 \_2$

American Psychiatric Association - APA (2013). Diagnostic and statistical manual of mental disorders (5th ed. -Text Revised). Washington, DC: APA.

Amir, N., Freshman, M., \& Foa, E. B. (2000). Family distress and involvement in relatives of obsessive-compulsive disorder patients. Journal of Anxiety Disorders, 14(3), 209-217. Retrieved from http://dx.doi.org/10.1016/S0887-6185(99)00032-8

Aron, A., Norman, C. C., Aron, E. N., McKenna, C., \& Heyman, R.E. (2000). Couples' shared participation in novel and arousing activities and experienced relationship quality. Journal 
of Personality and Social Psychology, 78(2), 273-284. Retrieved from http://psycnet.apa.org/journals/psp/78/2/273.html

Barker, C., \& Pistrang, N. (2002). Psychotherapy and social support: Integrating research on psychological helping. Clinical Psychology Review, 22(3), 361-379. Retrieved from http://eprints.ucl.ac.uk/2297/

Batinic, B., Trajkovic, G., Duisin, D., \& Nikolic-Balkoski, G. (2009). Life events and social support in a 1-year preceding panic disorder. Psychiatria Danubina, 21(1), 33-40. Retrieved from http://www.hdbp.org/psychiatria_danubina/pdf/dnb_vol21_no1/dnb_vol21_no1_33.pdf

Black, D. W., Gaffney, G., Schlosser, S., \& Gabel, J. (1998). The impact of obsessivecompulsive disorder on the family: Preliminary findings. Journal of Nervous and Mental Disease, 186, 440-442.

Brewin, C.R., Andrews, B., \& Valentine, J.D. (2000). Meta-analysis of risk factors for posttraumatic stress disorder in trauma-exposed adults. Journal of Consulting and Clinical Psychology, 68, 748-766. doi: 10.1037/0022-006X.68.5.748

Calvocoressi, L., Lewis, B., Harris, M., Trufan, S. J., Goodman, W. K., McDougle C. J., \& Price, L. H. (1995). Family accommodation in obsessive-compulsive disorder. American Journal of Psychiatry, 152(3), 441-3. Retrieved from http://ajp.psychiatryonline.org/article.aspx?articleid=170892

Calvocoressi, L., Mazure, C. M., Kasl, S. V., Skolnick, J., Fisk, D., Vegso, S. J., ... Price, L. H. (1999). Family accommodation of obsessive-compulsive symptoms: instrument development and assessment of family behavior. Journal of Nervous and Mental Disease, 187(10), 636-42. Retrieved from 
http://journals.lww.com/jonmd/Abstract/1999/10000/Family_Accommodation_of_Obsess ive_Compulsive.8.aspx

Chambless, D. L., Fauerbach, J. A., Floyd, F. J., Wilson, K. A., Remen, A. L., \& Renneberg, B. (2002). Marital interaction of agoraphobic women: a controlled, behavioral observation study. Journal of Abnormal Psychology, 111(3), 502-512. Retrieved from http://www.apa.org/pubs/journals/abn/index.aspx

CleveMed Research (2007). Bioradio 150 and Bioradio Capture Lite ${ }^{\mathrm{TM}}$ (version 1.02.07.0601) [Apparatus and software]. Cleveland, Ohio: CleveMed Medical Devices Inc. http://www.clevemed.com

Cohen, S., \& Wills, T. A. (1985). Stress, social support, and the buffering hypothesis. Psychological Bulletin, 98, 310-357. doi: 10.1037/0033-2909.98.2.310

Crevier, M. G., Marchand, A., Nachar, N., \& Guay, S. (2013). Overt social support behaviors: associations with PTSD, concurrent depressive symptoms and gender. Psychological Trauma: Theory, Research, Practice, and Policy, First Posting. doi: 10.1037/a0033193

Cutrona, C. E., \& Suhr, J. A. (1992). Controllability of stressful events and satisfaction with spouse support behaviors. Communication Research, 19, 154-174. doi: $10.1177 / 009365092019002002$

First, M.B., Spitzer, R.L., Gibbon, M., \& Williams, J.B.W. (1995). Structured clinical interview for Axis I DSM-IV disorders (Patient edition). New York: New York State Psychiatric Institute, Biometrics Research Department.

Foa, E.B., \& Rothbaum, B.O. (1998). Treating the trauma of rape: Cognitive behavioral therapy for PTSD. New York: Guilford Press. 
Foa, E.B., Steketee, G., \& Rothbaum, B.O. (1989). Behavioral/cognitive conceptualizations of post-traumatic stress disorder. Behavior Therapy, 20, 155-176. Retrieved from http://dx.doi.org/10.1016/S0005-7894(89)80067-X

Foy, D.W., Osato, S.S., Houskamp, B.M., \& Neumann, D.A. (1992). Etiology of posttraumatic stress disorder. In P.A. Saigh (Ed.), Posttraumatic stress disorder (pp. 28-49). New York: Macmillan.

g.BSanalyze software (version 3.07a) [Computer software]. Graz, Autriche: Guger technologies. http://www.gtec.at

Guay, S., Beaulieu-Prévost, D. Beaudoin, C., St-Jean Trudel, É., Nachar, N., Marchand, A., and O'Connor, K. (2011). How do social interactions with a significant other affect PTSD symptoms? An empirical investigation using a clinical sample. Journal of Aggression, Maltreatment \& Trauma, 20(3), 280-303. doi: 10.1080/10926771.2011.562478

Guay, S., Billette, V., \& Marchand, A. (2006). Exploring the links between post-traumatic stress disorder and social support : Processes and potential research avenues. Journal of Traumatic Stress, 19, 1-12. doi: 10.1002/jts.20124

Hopper, J. W., Spinazzola, J., Simpson, W. B., \& van der Kolk, B. A. (2006). Preliminary evidence of parasympathetic influence on basal heart rate in posttraumatic stress disorder. Journal of Psychosomatic Research, 60(1), 83-90. Retrieved from http://dx.doi.org/10.1016/j.jpsychores.2005.06.002

Horowitz, M.J. (1986). Stress response syndrome (2 ${ }^{\text {nd }}$ ed.). New York: Jason Aronson.

Hou, S. Y., Yen, C. F., Huang, M. F., Wang, P. W., \& Yeh, Y. C. (2010). Quality of life and its correlates in patients with obsessive-compulsive disorder. Kaohsiung Journal of Medical Science, 26(8), 397-407. doi: 10.1016/S1607-551X(10)70065-6 
Jones, J.C., \& Barlow, D.H. (1990). The etiology of post-traumatic disorder. Clinical Psychology Review, 10, 299-328.

Joseph, S., Williams, R., \& Yule, W. (1997). Understanding post-traumatic stress: A psychosocial perspective on PTSD and treatment. Wiley: Chichester, England.

Keane, T. M., Zimering, R. T., \& Caddell, J. M. (1985). A behavioral formulation of posttraumatic stress disorder in combat veterans. The Behavior Therapist, 8, 9-12.

Laffaye, C., Cavella, S., Drescher, K., \& Rosen, C. (2008). Relationship among PTSD symptoms, social support, and support sources in veterans with chronic PTSD. Journal of Traumatic Stress, 4, 394-401. doi: 10.1002/jts.20348

Lakey, B., \& Cohen, S. (2000). Social support theory and measurement. In S. Cohen, L.G. Underwood, \& B.H. Gottlieb (Eds.), Social support measurement and intervention (pp. 29-52). New York: Oxford University Press.

Lang, P. J., \& McTeague, L. M. (2009). The anxiety disorder spectrum: Fear imagery, physiological reactivity, and differential diagnosis. Anxiety Stress and Coping, 22(1), 525. doi: $10.1080 / 10615800802478247$

McTeague, L.M., Lang, P.J., Laplante, M.C., Cuthbert, B.N., Shumen, J.R., \& Bradley, M.M. (2010). Aversive imagery in posttraumatic stress disorder: Trauma recurrence, comorbidity, and physiological reactivity. Biological Psychiatry, 67, 346-56. doi: 10.1016/j.biopsych.2009.08.023

McTeague, L.M., Lang, P.J., Laplante, M.C., Cuthbert, B.N., Strauss, C.C., \& Bradley, M.M. (2009). Fearful imagery in social phobia: Generalization, comorbidity, and physiological reactivity. Biological Psychiatry, 65, 374-382. doi: 10.1016/j.biopsych.2008.09.023

Nachar, N., Lavoie, M.E., Marchand, A., O’Connor, K.P., \& Guay, S. (2014). The effect of talking about psychological trauma with a significant other on heart rate reactivity in 
individuals with posttraumatic stress disorder. Psychiatry Research, 219(1), 171-176. doi: http://dx.doi.org/10.1016/j.psychres.2014.05.006

Ozer, E.J., Best, S.R., Lipsey, T.L., \& Weiss D.S. (2003). Predictors of post-traumatic stress disorder and symptoms in adults: A meta-analysis. Psychological Bulletin, 129, 52-73. doi: $10.1037 / 0033-2909.129 .1 .52$

Pash, L.A., \& Bradbury, T. N. (1998). Social support, conflict, and the development of marital dysfunction. Journal of Consulting and Clinical Psychology, 66, 219-230. doi: 10.1037/0022-006X.66.2.219

Pole, N. (2006). Moderators of PTSD-related psychophysiological effect sizes: Results from a meta-analysis. Annals of the New York Academy of Sciences, 1071(1), 422-4. doi: 10.1196/annals. 1364.033

Pole, N. (2007). The psychophysiology of posttraumatic stress disorder: A meta-analysis. Psychological Bulletin, 133(5), 725-746. doi: 10.1037/0033-2909.133.5.725

Pyke, J., \& Roberts, J. (1987). Social support and married agoraphobic women. Canadian Journal of Psychiatry, 32(2), 100-104. Retrieved from http://www.ncbi.nlm.nih.gov/pubmed/3567817

Renshaw, K.D., Chambless, D. L., \& Steketee, G. (2003). Perceived criticism predicts severity of anxiety symptoms after behavioral treatment in patients with obsessive-compulsive disorder and panic disorder with agoraphobia. Journal of Clinical Psychology, 59(4), 41121. doi : $10.1002 /$ jclp. 10048

Sack, M., Hopper, J. W., \& Lamprecht, F. (2004). Low respiratory sinus arrhythmia and prolonged psychophysiological arousal in posttraumatic stress disorder: heart rate dynamics and individual differences in arousal regulation. Biological Psychiatry, 55(3), 284-290. Retrieved from http://dx.doi.org/10.1016/S0006-3223(03)00677-2 
Sharpley, C.F. (1993). Effects of brief rest periods upon heart rate in multiple baseline studies of heart rate reactivity. Biofeedback Self-Regulation, 18(4), 225-35.

Stein, P.K., \& Boutcher, S.H. (1993). Heart-rate and blood-pressure responses to speech alone compared with cognitive challenges in the stroop task. Perception and Motor Skills, 77, 555-563. http://psycnet.apa.org/doi/10.2466/pms.1993.77.2.555

Steketee, G.S. (1993). Treatment of obsessive compulsive disorder. New York: Guilford Press.

Uchino, B.N., Cacioppo, J.T., \& Kiecolt-Glaser, J.K. (1996). The relationship between social support and physiological processes: A review with emphasis on underlying mechanisms and implications for health. Psychological Bulletin, 119, 488-531. Retrieved from http://www.apa.org/pubs/journals/bul/index.aspx

Ullman, S. E., \& Filipas, H. H. (2001). Predictors of PTSD symptom severity and social reactions in sexual assault victims. Journal of Traumatic Stress, 14(2), 369-389.

doi: $10.1023 / \mathrm{A}: 1011125220522$

Williams, R., \& Joseph, S. (1999). Conclusions: An integrative psychosocial model of PTSD. In W. Yule (Ed.), Post-traumatic stress disorders: Concepts and therapy (pp. 297-314). Chichester, England: Wiley.

Wills, T.A., \& Shiner, O. (2000). Measuring perceived and received social support. In S. Cohen, L.G. Underwood, \& B.H. Gottlieb (Eds.), Social support measurement and intervention (pp. 86-135). New York: Oxford University Press.

Word count: 7,122 words (Including References, Excluding Abstract and Tables) 